\title{
POTENCIAL PRODUTIVO E QUALIDADE DE FRUTOS DE MACIEIRAS TRATADAS COM GIBERELINA E INIBIDOR DA BIOSSÍNTESE DE GIBERELINAS ${ }^{1}$
}

\author{
JOÃO PAULO GENEROSO SILVEIRA ${ }^{2}$, CASSANDRO VIDAL TALAMINI DO AMARANTE ${ }^{3}$, \\ CRISTIANO ANDRÉ STEFFENS ${ }^{4}$, THAIS ROSELI CORREA ${ }^{2}$, FRANCIELLE NETO PAES ${ }^{5}$
}

RESUMO - Trabalhos recentes mostram que a pulverização de macieiras com inibidor da biossíntese de giberelinas pode reduzir o crescimento vegetativo das plantas e melhorar a qualidade dos frutos. O objetivo deste trabalho foi avaliar os efeitos da pulverização de macieiras com um inibidor da biossíntese de giberelinas, prohexadiona-cálcio (ProCa), e com giberelina $\left(\mathrm{GA}_{3}\right)$, sobre a floração, frutificação e qualidade dos frutos. O experimento foi conduzido em pomar localizado no município de São Joaquim-SC, na safra de 2009/2010. Macieiras 'Catarina' e 'Fuji' foram pulverizadas com água (tratamento- controle), ProCa e $\mathrm{GA}_{3}$ [ambos os produtos na dose de $319 \mathrm{~g}$ (i.a.) ha' ${ }^{-1}$, quando as brotações do ano estavam com 5-10 cm de comprimento, sendo repetidas após 20 dias. A contagem do número de cachos florais e de frutos nas plantas ocorreram, respectivamente, nos meses de outubro e novembro, em 2009 e 2010 . Os frutos foram submetidos às análises de qualidade na colheita e após quatro meses de armazenamento refrigerado $\left(0 \pm 0,5^{\circ} \mathrm{C} / 90-95 \%\right.$ UR), seguido de sete dias de comercialização simulada $\left(20 \pm 4{ }^{\circ} \mathrm{C} / 60-70 \%\right.$ UR). No ano subsequente ao da aplicação dos tratamentos (2010), macieiras 'Fuji' pulverizadas com ProCa apresentaram menor frutificação do que o tratamentocontrole. O tratamento com ProCa proporcionou maior coloração vermelha em maçãs 'Catarina'. No momento da colheita, maçãs 'Fuji' e 'Catarina' provenientes de plantas pulverizadas com ProCa apresentaram maior força para a penetração da polpa na região mais vermelha dos frutos. Após o armazenamento, maçãs 'Fuji' de plantas pulverizadas com $\mathrm{GA}_{3}$ apresentaram menor firmeza de polpa e maçãs 'Catarina' de plantas pulverizadas com ProCa apresentaram maior firmeza de polpa. A pulverização de $\mathrm{GA}_{3}$ em macieiras, em pós-floração (duas aplicações, cada aplicação na dose de $319 \mathrm{~g} \mathrm{ha}^{-1}$ ), pode comprometer a floração no ano subsequente à sua aplicação, bem como ocasionar a alteração em alguns dos atributos de qualidade dos frutos, indicando um avanço na maturação. O ProCa pode aumentar a firmeza de polpa e a porcentagem de cor vermelha em frutos de macieiras 'Catarina', e reduzir a floração e a frutificação no ano subsequente à sua aplicação em macieiras 'Fuji'.

Termos de indexação: Malus domestica Borkh, prohexadiona-cálcio, antigiberelina, frutos, firmeza de polpa.

\section{YIELD POTENTIAL AND FRUIT QUALITY OF APPLE TREES TREATED WITH GIBBERRELLIN AND INHIBITOR OF GIBBERELLINS BIOSYNTHESIS}

\begin{abstract}
Recently published study has shown the apple trees treated with inhibitor of gibberellins biosynthesis have reduced vegetative growth and improved fruit quality. The aim of this study was to evaluate effects of spraying apple trees with prohexadione-calcium (ProCa) (an inhibitor of gibberellins biosynthesis) and gibberellin ( $\left(\mathrm{GA}_{3}\right)$ on yield potential and fruit quality. The experiment was conducted in an orchard located in São Joaquim, State of Santa Catarina (Southern Brazil) in 2009/2010. 'Catarina' and 'Fuji' apple trees were treated with water (control), ProCa and $\mathrm{GA}_{3}$ [both products at the dose of $319 \mathrm{~g}$ (a.i.) ha ${ }^{-1}$, when shoots were 5-10 cm long, with treatments repeated after 20 days. The number of flower buds and fruits in the plants was assessed in October and November, respectively, in 2009 and 2010 . Fruit quality was assessed at harvest and after four months of cold storage $\left(0 \pm 0.5{ }^{\circ} \mathrm{C} / 90-95 \% \mathrm{RH}\right)$, followed by seven days of simulated marketing $\left(20 \pm 4{ }^{\circ} \mathrm{C} / 60-70 \% \mathrm{RH}\right)$. In the year following the treatment of the trees (2010), 'Fuji' apples treated with ProCa had lower fruit set than the control. ProCa increased the red color in 'Catarina' apples. At harvest, the force for flesh penetration in the red side of the fruit was increased in apple trees of both cultivars treated with ProCa. After cold storage, 'Fuji' apples of trees treated with $\mathrm{GA}_{3}$ had lowest flesh firmness, while 'Catarina' apples of trees treated with ProCa had highest flesh firmness. The GA sprayed after full bloom (two treatments, each one at $319 \mathrm{~g} \mathrm{ha}^{-1}$ ) might reduce the bloom of the following season and cause some changes in fruit quality attributes, leading to advanced maturity. The treatment with ProCa may increase the flesh firmness and red color in the 'Catarina' apples, and reduce return bloom and fruit set in the year following the treatment in 'Fuji' apples.

Index terms: Malus domestica Borkh, prohexadione-calcium, anti-gibberellin, fruit, flesh firmness.

${ }^{1}$ (Trabalho 352-13). Recebido para publicação em: 25-09-2013. Aceito para publicação em: 20-08-2014.

${ }^{2}$ Mestrandos em Produção Vegetal, CAV/UDESC. Lages-SC. E-mail: joaop-silveira@hotmail.com; thaisroselicorrea@hotmail.com ${ }^{3}$ Ph.D., Bolsista do CNPq, Prof. do Depto. de Agronomia, CAV/UDESC. Lages, SC. E-mail: amarante@cav.udesc.br ${ }^{4}$ Dr., Professor do Depto. de Agronomia, CAV/UDESC. Lages-SC. E-mail: steffens@cav.udesc.br

${ }^{5}$ Graduanda do Curso de Agronomia, Bolsista FAPESC. CAV/UDESC. Lages-SC. E-mail: francielle.netopaes@hotmail.com
\end{abstract}




\section{INTRODUÇÃO}

A cultura da macieira (Malus domestica Borkh.) é de grande importância econômica para o Estado de Santa Catarina, que é responsável por 60\% da produção nacional (PEREIRA et al., 2010). Todavia, esta cultura ainda apresenta grandes problemas relacionados às perdas pós-colheita de frutos, ocasionadas por alterações fisiológicas associadas à maturação e senescência (AMARANTE et al., 2010).

A utilização de reguladores vegetais pode retardar a maturação e a colheita de frutos, além de reduzir as perdas durante o armazenamento (STEFFENS et al., 2009). Dentre estes, o ácido giberélico destaca-se por proporcionar a preservação da qualidade pós-colheita de frutíferas de clima temperado, como o pêssego e a ameixa (AMARANTE et al., 2005; STEFFENS et al., 2009). Os efeitos da aplicação de $\mathrm{GA}_{4+7}$ em pós-floração para a maturação de frutos de macieira são dependentes da cultivar, apresentando, por exemplo, efeito nulo na cv. Cameo e acelerando a maturação na cv. Honeycrisp (SCHMIDT et al., 2008).

Outro regulador potencial que pode favorecer a qualidade e a maturação de frutos é o retardante prohexadiona-cálcio (ProCa). Este inibidor da biossíntese de giberelinas é apontado como alternativa para o controle do crescimento vegetativo em macieiras (BASAK, 2004; COSTA et al., 2004; HAWERROTH et al., 2012a) e pereiras (HAWERROTH et al., 2012b). Verifica-se que a aplicação em pré-colheita do ProCa reduz o crescimento vegetativo e, com isso, diminui a competição entre ramos e frutos pelos fotoassimilados disponíveis, podendo resultar em melhor qualidade dos frutos (ZHANG; WHITING, 2011). A pulverização de macieiras com ProCa após a floração proporcionou aumento na intensidade e na porcentagem de cor vermelha em maçãs 'Fuji' (MEDJDOUB et al., 2005) e na firmeza de polpa em maçãs 'McIntosh' (GREENE, 1999). Segundo HAWERROTH et al. (2012), aplicações de ProCa, na dose de $330 \mathrm{~g}$ (i.a) ha ${ }^{-1}, 20$ a 28 dias após a plena floração, reduzem o comprimento médio e a massa fresca média dos ramos podados em macieiras 'Imperial Gala' cultivadas no Sul do Brasil. Porém, a utilização de doses altas deste produto pode reduzir a massa dos frutos, além de comprometer a floração do ciclo seguinte (GREENE, 2008).

Diante do exposto acima, objetivou-se avaliar os efeitos da pulverização em pós-floração de macieiras 'Catarina' e 'Fuji' com prohexadiona cálcio e ácido giberélico, sobre o potencial produtivo das plantas e a qualidade dos frutos.

\section{MATERIAL E MÉTODOS}

O experimento foi conduzido em um pomar localizado no município de São Joaquim- SC (latitude $28^{\circ} 16^{\prime} 40,02^{\prime \prime} \mathrm{S}$, longitude $49^{\circ} 56^{\prime} 09,10$ ' W e altitude de $1.400 \mathrm{~m}$ ), nas safras de 2009 e 2010. Foram utilizadas macieiras 'Catarina' (14 anos de idade, sobre porta-enxerto 'Marubakaido' e espaçamento de $2 \mathrm{~m}$ entre plantas e $6,5 \mathrm{~m}$ entre filas) e 'Fuji' (13 anos de idade, sobre porta-enxerto 'Marubakaido' com filtro M9 e espaçamento de 1,5 m entre plantas e 4,5 m entre filas), conduzidas com líder central. As macieiras 'Catarina' e 'Fuji' foram pulverizadas com água (tratamento-controle), prohexadiona-cálcio [ProCa; 319 g (i.a.) ha ha $^{-1}$ e giberelina $\left[\mathrm{GA}_{3} ; 319\right.$ g (i.a.) ha ${ }^{-1}$, na queda das pétalas, quando as brotações do ano estavam com $5-10 \mathrm{~cm}$ de comprimento, e novamente 20 dias após a $1^{\mathrm{a}}$ aplicação. Foi utilizado um volume de calda de $1.000 \mathrm{~L} \mathrm{ha}^{-1}$, procurando atingir o completo molhamento foliar da planta.

Os dados de cada cultivar foram analisados de forma independente. Em ambas as cultivares, foi utilizado o delineamento experimental em blocos ao caso, com cinco repetições, sendo cada repetição constituída de uma planta.

Nos dois anos de avaliação, realizou-se a contagem do número de cachos florais, em outubro, e de frutos, em novembro, em ambas as cultivares. A partir da determinação do diâmetro dos ramos, obteve-se a proporção de cachos florais e frutos por $\mathrm{cm}^{2}$ de secção transversal dos ramos.

$\mathrm{Na}$ colheita, foram separadas amostras contendo 50 frutos por repetição, para ambas as cultivares. Uma subamostra de 20 frutos por repetição foi submetida às seguintes análises: percentagem de cor vermelha e atributos de cor da epiderme, massa do fruto inteiro, atributos de textura, firmeza de polpa, acidez titulável (AT), sólidos solúveis (SS) e teste de iodo-amido. Outra subamostra de 30 frutos por repetição foi armazenada em câmara fria convencional $\left(0 \pm 0,5{ }^{\circ} \mathrm{C} / 90-95 \%\right.$ UR), durante quatro meses, seguido de sete dias de comercialização simulada $\left(20 \pm 4{ }^{\circ} \mathrm{C} / 60-70 \%\right.$ UR), e após este período, submetidas às mesmas avaliações efetuadas na colheita, exceto quanto ao índice de iodo-amido e percentagem de cor vermelha da casca.

A percentagem de cor vermelha foi determinada por meio de análise subjetiva da superfície dos frutos recoberta com cor vermelha. A determinação da cor da epiderme foi efetuada com colorímetro Minolta, modelo CR 400, sendo 
as leituras realizadas nas regiões mais e menos vermelhas do fruto, correspondendo às regiões mais e menos expostas à radiação, e os resultados expressos nos atributos $L, C$ e $h^{\circ}$. O $h^{\circ}$ (ângulo hue) define a coloração básica, sendo que $0^{\circ}=$ vermelho, $90^{\circ}=$ amarelo e $180^{\circ}=$ verde. $\mathrm{O} L$ (ligthness) define a luminosidade, que varia de zero (preto) a 100 (branco). O C define a cromaticidade (quanto maior, mais intensa é a definição de cor).

Os atributos de textura da epiderme e da polpa foram analisados com texturômetro eletrônico TAXT-plus $^{\circledR}$ (Stable Micro Systems Ltd., Reino Unido). Para a quantificação, foi utilizada ponteira modelo PS2, com $2 \mathrm{~mm}$ de diâmetro, a qual foi introduzida na polpa a uma profundidade de $10 \mathrm{~mm}$, com velocidades pré-teste, teste e pós-teste de $30 ; 5$ e $30 \mathrm{~mm} \mathrm{~s}^{-1}$, respectivamente.

A firmeza de polpa $(\mathrm{N})$ foi determinada com o auxílio de penetrômetro, equipado com ponteira de 11 mm de diâmetro, em duas regiões opostas, na porção equatorial dos frutos, após remoção da epiderme.

Os valores de AT (\% de ácido málico) foram obtidos por meio de uma amostra de $10 \mathrm{~mL}$ de suco dos frutos, obtidos por meio de uma centrífuga modelo RI 6720. Esta amostra foi diluída em $90 \mathrm{~mL}$ de água destilada e titulada com solução de $\mathrm{NaOH}$ $0,1 \mathrm{~N}$ até $\mathrm{pH} 8,1$.

Os teores de $\mathrm{SS}$ ( ${ }^{\circ}$ Brix) foram determinados com refratômetro digital, modelo PR201 $\alpha$ (Atago, Tóquio, Japão), utilizando-se do suco extraído conforme descrito para AT.

$\mathrm{O}$ teste de iodo-amido foi determinado por meio da comparação do escurecimento da metade peduncular dos frutos tratada com solução de iodo, em uma escala de 1 a 5 , em que o índice 1 indica o teor máximo de amido e o índice 5 representa o amido totalmente hidrolisado.

Inicialmente, verificaram-se os dados quanto ao atendimento dos pressupostos da análise de variância, por meio da aplicação dos testes de Barlett (homogeneidade) e Shapiro-Wilk (normalidade). Esses requisitos foram atendidos, e os dados não foram transformados. A significância dos efeitos dos tratamentos foi determinada pelo teste $\mathrm{F}$, sendo as médias comparadas pelo teste de Tukey $(\mathrm{p}<0,05)$, em cada cultivar, utilizando o programa estatístico SAS (SAS INSTITUTE, 2002).

\section{RESULTADOS E DISCUSSÃO}

No primeiro ano de avaliação (2009), a aplicação de reguladores de crescimento não alterou a floração (número de cachos florais $/ \mathrm{cm}^{2}$ de seção transversal de ramo) das cultivares Catarina e Fuji (Tabela 1). Porém, no ano subsequente (2010), ambas as cultivares apresentaram redução na floração quando tratadas com $\mathrm{GA}_{3}$ (Tabela 1). Este mesmo resultado foi observado em macieiras 'York' ao receberem $\mathrm{GA}_{4+7}$ em pós-floração (DAVIS, 2002). Em plantas perenes, o $\mathrm{GA}_{3}$ geralmente inibe o florescimento (WILKIE et al., 2008; GOTTGENS; HEDDEN, 2009). Este comportamento pode estar relacionado ao efeito das giberelinas na promoção do crescimento vegetativo das plantas. $\mathrm{O} \mathrm{GA}_{3}$ pode inibir a indução floral pelo maior aporte de fotoassimilados para as gemas vegetativas da parte aérea, que crescem rapidamente (GOTTGENS; HEDDEN, 2009), com isso reduzindo a floração no ano subsequente ao de sua aplicação.

A pulverização com $\mathrm{GA}_{3}$ reduziu a frutificação no primeiro ano (2009) de aplicação dos tratamentos apenas em macieiras 'Fuji' (Tabela 1). De acordo com Davis (2002), macieiras 'Braeburn' tratadas em pós-floração com $\mathrm{GA}_{3}$ ou $\mathrm{GA}_{4+7}$ apresentaram redução na frutificação de gemas laterais, nas regiões proximal e mediana dos ramos. Entretanto, segundo este mesmo autor, macieiras 'York' submetidas ao mesmo tratamento e época de aplicação apresentaram frutificação similar ao tratamento-controle. Desta forma, é possível observar a diferença de comportamento entre as cultivares de macieiras pulverizadas com GAs, o que foi observado no presente trabalho para as cultivares Fuji e Catarina.

A dose de ProCa utilizada (319 $\mathrm{g} \mathrm{ha}^{-1}$ ) reduziu a floração nas macieiras 'Fuji', bem como comprometeu a frutificação, no ano seguinte ao da aplicação do produto (2010), em relação ao tratamento-controle (Tabela 1). Em macieiras 'McIntosh', no ano seguinte ao da aplicação de ProCa (250 $\mathrm{mg} \mathrm{L}^{-1}$ ), foi possível observar redução tanto na floração quanto na frutificação (GREENE, 2008). No entanto, ProCa (42 $\mathrm{mg} \mathrm{L}^{-1}$ ) foi eficiente no controle do crescimento vegetativo, sem comprometer a floração do ciclo seguinte em macieiras 'Mutsu' (GREENE, 2008). Em Fraiburgo-SC, Hawerroth et al. (2012a) verificaram que ProCa, aplicado em diferentes épocas (20 a 200 DAPF), na dose total de $330 \mathrm{~g} \mathrm{ha}^{-1}$, não reduziu a produção de fruto por planta em macieiras 'Imperial Gala' enxertadas sobre 'Marubakaido' com interenxerto 'M9'. Diferenças observadas nos resultados obtidos por outros autores 
podem ser devido a efeitos de clima e/ou cultivares de macieiras. Além disso, isto pode estar relacionado à maior dose utilizada neste trabalho (foram duas aplicações do ProCa na dose de $319 \mathrm{~g} \mathrm{ha}^{-1}$, resultando numa dose total de $638 \mathrm{~g} \mathrm{ha}^{-1}$ ), enquanto Hawerroth et al. (2012a) utilizaram apenas uma aplicação do produto, na dose de $330 \mathrm{~g} \mathrm{ha}^{-1}$. Desta forma, verificase que a otimização do uso de ProCa depende do estabelecimento de dose para cada cultivar avaliada e região de produção.

Plantas do tratamento-controle apresentavam, na colheita, firmeza de polpa de $85-90 \mathrm{~N}$ para 'Catarina' e de $75-80 \mathrm{~N}$ para 'Fuji', correspondendo à maturação comercial, estádio normalmente utilizado pelos produtores para a colheita das duas cultivares na região de São Joaquim-SC.

A aplicação de $\mathrm{GA}_{3}$ em macieiras 'Catarina' e 'Fuji' propiciou os maiores valores de $L$ e $C$ na região dos frutos mais exposta à radiação (lado mais vermelho) (Tabela 2). Estes atributos indicam um vermelho menos intenso (maior brilho) e mais saturado. Este resultado pode estar relacionado ao efeito do $\mathrm{GA}_{3}$ em aumentar o crescimento vegetativo (SILVEIRA et al., 2012), reduzindo a exposição do fruto à radiação. Isto diminui a biossíntese de antocianinas e a intensidade de cor vermelha nos frutos (AMARANTE et al., 2005; STEFFENS et al., 2009). O atributo de cor $h^{\circ}$ na região de menor exposição à luz (lado menos vermelho) foi menor em maçãs 'Catarina' e 'Fuji' tratadas com $\mathrm{GA}_{3}$ (Tabela 2). De acordo com Zhang et al. (2008), a pera 'Japonesa' tratada em pré-colheita com $\mathrm{GA}_{4}$ ou $\mathrm{GA}_{7}$ apresenta antecipação da maturação, em virtude da redução no conteúdo de clorofila na epiderme dos frutos, indicada pelos menores valores de $h^{\circ}$ na casca. O mesmo pode ter acontecido com maçãs 'Catarina' e 'Fuji' tratadas com $\mathrm{GA}_{3}$ (Tabela 2).

No momento da colheita, maçãs 'Catarina' colhidas de plantas tratadas com ProCa apresentaram os maiores valores de porcentagem de cor vermelha (Tabela 2). Resultados semelhantes foram reportados por Medjdoub et al. (2005). Isto mostra relação inversa entre vigor vegetativo e desenvolvimento de cor vermelha no fruto. $\mathrm{O}$ menor crescimento vegetativo em macieiras pulverizadas com ProCa (SILVEIRA et al., 2012) pode aumentar a exposição dos frutos à radiação solar. Portanto, nestas condições, pode haver maior síntese de antocianinas na epiderme dos frutos (AMARANTE et al., 2007), resultando no maior desenvolvimento de cor vermelha (Tabela 2). Maçãs 'Catarina' também apresentaram menores valores de $L$ na região com maior exposição à luz (região mais vermelha) quando tratadas com ProCa (Tabela 2). Mesmo na região dos frutos menos exposta à radiação (lado menos vermelho), houve redução nos valores de $L$ em maçãs 'Catarina' e 'Fuji' colhidas de plantas tratadas com ProCa (Tabela 2). Da mesma forma, Medjdoub et al. (2005) observaram menores valores de $L$ na região mais exposta à luz em maçãs provenientes de plantas tratadas com ProCa, indicando vermelho mais intenso na epiderme dos frutos.

Na colheita, maçãs 'Catarina' provenientes de plantas pulverizadas com $\mathrm{GA}_{3}$ apresentaram menor AT do que o tratamento-controle (Tabela 3). Em maçãs 'Fuji' submetidas ao mesmo tratamento, observaram-se o maior teor de SS e os menores valores de força para ruptura da epiderme, tanto no lado mais vermelho quanto no menos vermelho, e de força para penetração da polpa no lado mais vermelho dos frutos (Tabela 3). Os demais atributos de qualidade não diferiram entre os tratamentos com $\mathrm{GA}_{3}$ e controle, em maçãs 'Catarina' e 'Fuji' (Tabela 3). Schmidt et al. (2008) observaram efeito linear do aumento na dose de $\mathrm{GA}_{4+7}$ em pós-floração, na degradação de amido e na redução na firmeza e AT, em maçãs 'Honeycrisp', sugerindo antecipação na maturação de dois a cinco dias em relação aos frutos do tratamento-controle. Desta forma, a dose de $\mathrm{GA}_{3}\left(319 \mathrm{~g} \mathrm{ha}^{-1}\right)$ utilizada pode corresponder a um nível elevado e prejudicial aos frutos, resultando no avanço de alguns atributos de maturação em maçãs ‘Catarina' e 'Fuji' (Tabela 3), ocasionado pelo aumento na produção de etileno (SCHMIDT et al., 2008). Adicionalmente, segundo De Grauwe et al. (2008), giberelinas podem aumentar a estabilidade da enzima ACC sintase, e com isso aumentar a biossíntese de etileno.

Na colheita, maçãs 'Catarina' de plantas tratadas com ProCa apresentaram menor AT e maior força para penetração da polpa na região mais vermelha dos frutos (Tabela 3). Em maçãs 'Fuji' provenientes do mesmo tratamento, foram observados maiores valores de AT e de força para penetração da polpa na região mais vermelha dos frutos (Tabela 3). De acordo com Greene (1999), maçãs 'McIntosh' de plantas submetidas ao tratamento com ProCa após a floração também apresentaram aumento na firmeza de polpa na colheita comercial. Khurshid et al. (1997) também verificaram aumento na firmeza de frutos de maçãs oriundas de plantas tratadas com outro inibidor da biossíntese de ácido giberélico, o Paclobutrazol.

A pulverização das plantas com ProCa após a floração ocasiona a redução no crescimento vegetativo das plantas (SILVEIRA et al., 2012), com isso podendo aumentar a exposição dos frutos à luminosidade. Desta forma, nessas condições, pode ocorrer a modificação na composição da parede 
celular, no número de células, ou no turgor celular na região do fruto mais exposta ao sol (FERGUSON et al., 1999), com isso, podendo resultar no aumento da firmeza de polpa nesta região dos frutos. Segundo Yeshitela et al. (2004), a supressão do crescimento vegetativo em plantas lenhosas tratadas com inibidores da síntese de giberelinas favorece o aporte de fotoassimilados para o fruto em desenvolvimento, podendo assim melhorar alguns dos seus atributos de qualidade, principalmente o aumento na firmeza de polpa.

Após o período de armazenamento, maçãs 'Fuji' provenientes de plantas pulverizadas com $\mathrm{GA}_{3}$ apresentaram maior teor de SS, menores valores de firmeza de polpa e de forças para a ruptura da epiderme e para a penetração da polpa na região mais vermelha dos frutos (Tabela 4). A pulverização de $\mathrm{GA}_{4}$ ou GA ${ }_{4+7}$ em pós-floração, na dose de $400 \mathrm{mg} \mathrm{L}^{-1}$, em macieiras 'Honeycrisp', também proporcionou redução na firmeza dos frutos após 140 dias de armazenamento refrigerado (SCHMIDT et al., 2008). Este comportamento pode estar relacionado ao avanço na maturação, observado no momento da colheita, proporcionado pelas altas doses de GA. Isto pode refletir no avanço do amadurecimento dos frutos em pós-colheita. Entretanto, para a cultivar Cameo, a pulverização de $\mathrm{GA}_{4+7}$ nas doses de 300; 600; 900 e $1.200 \mathrm{mg} \mathrm{L}^{-1}$ não proporcionou nenhum efeito nos atributos de maturação dos frutos, tanto na colheita como após 120 dias de armazenamento refrigerado (SCHMIDT et al., 2008). Isto mostra a diferença de resposta entre cultivares de macieiras ao tratamento com giberelinas.

Em frutos proveniente de macieiras 'Catarina' tratadas com ProCa foi possível observar melhor manutenção da firmeza de polpa e da força para a ruptura da epiderme na região mais vermelha do fruto, após o armazenamento (Tabela 4). De acordo com Greene (1999), maçãs ‘McIntosh' provenientes de plantas pulverizadas com ProCa apresentaram maior firmeza de polpa após o armazenamento, com isso aumentando a vida pós-colheita dos frutos. Macieiras tratadas com ProCa apresentaram maior teor de Ca nos frutos, o que contribui para preservar a firmeza de polpa e reduzir a ocorrência de "bitter pit" (distúrbio fisiológico associado à deficiência de Ca nos frutos) em pós-colheita (SILVEIRA et al., 2012). Frutos com "bitter pit" apresentam baixa integridade de membrana plasmática e alta perda de água, o que resulta na redução da pressão de turgor da célula, ocasionando a perda de firmeza e textura (FREITAS et al., 2010).

$\mathrm{O}$ aumento na porcentagem de cor vermelha em maçãs 'Catarina' e na consistência de polpa, principalmente na região mais vermelha dos frutos, em ambas as cultivares pulverizadas com ProCa, mostra que este produto pode ser nova alternativa para os pomicultores da região. No entanto, sua aplicação pode inibir o processo de diferenciação floral e, assim, comprometer a produção na safra posterior à de sua aplicação. Portanto, são necessários novos trabalhos, testando diferentes doses e épocas de aplicação, para avaliar melhor os seus efeitos no potencial produtivo das plantas e na qualidade dos frutos na região Sul do Brasil. 
TABELA 1- Floração e frutificação, nos anos de 2009 e 2010, em macieiras 'Fuji' e 'Catarina' submetidas aos tratamentos-controle, com prohexadiona-cálcio (ProCa) e giberelina $\left(\mathrm{GA}_{3}\right)$.

\begin{tabular}{|c|c|c|}
\hline Tratamento & Floração $\left(\mathrm{n}^{\circ}\right.$ de cachos florais $\left./ \mathrm{cm}^{2}\right)$ & Frutificação $\left(\mathrm{n}^{\circ}\right.$ de frutos $\left./ \mathrm{cm}^{2}\right)$ \\
\hline & & 009 \\
\hline & & \\
\hline Controle & $2,66 \mathrm{a}$ & $2,45 \mathrm{a}$ \\
\hline ProCa & $2,66 \mathrm{a}$ & $3,01 \mathrm{a}$ \\
\hline $\mathrm{GA}_{3}$ & $2,74 a$ & $1,51 \mathrm{a}$ \\
\hline $\mathrm{CV}^{3}(\%)$ & 21,3 & 38,0 \\
\hline & & \\
\hline Controle & $5,44 a$ & $7,77 \mathrm{a}$ \\
\hline ProCa & $5,65 a$ & $7,44 a$ \\
\hline $\mathrm{GA}_{3}$ & $5,32 \mathrm{a}$ & $4,98 b$ \\
\hline CV $(\%)$ & 26,5 & 36,5 \\
\hline & AN & \\
\hline & & \\
\hline Controle & $7,27 \mathrm{a}$ & $5,22 \mathrm{a}$ \\
\hline ProCa & $7,42 \mathrm{a}$ & $2,38 \mathrm{a}$ \\
\hline $\mathrm{GA}_{3}$ & $1,33 b$ & $3,69 a$ \\
\hline $\mathrm{CV}^{3}(\%)$ & 23,1 & 24,0 \\
\hline & & \\
\hline Controle & $7,03 \mathrm{a}$ & $12,1 \mathrm{a}$ \\
\hline ProCa & $2,36 b$ & $3,98 b$ \\
\hline $\mathrm{GA}_{3}$ & $1,99 b$ & $8,80 \mathrm{ab}$ \\
\hline $\mathrm{CV}^{3}(\%)$ & 24,6 & 33,2 \\
\hline
\end{tabular}

Médias seguidas de mesma letra, nas colunas, para uma mesmo ano de avaliação e cultivar, não diferem entre si, pelo teste de Tukey $(\mathrm{p}<0,05)$.

TABELA 2- Cor vermelha (\%) e atributos de cor $\left(L, C\right.$ e $\left.h^{\circ}\right)$ da epiderme em maçãs 'Fuji' e 'Catarina', colhidas de plantas submetidas aos tratamentos-controle, com prohexadiona-cálcio (ProCa) e giberelina $\left(\mathrm{GA}_{3}\right)$.

\begin{tabular}{|c|c|c|c|c|c|c|c|}
\hline \multirow[t]{2}{*}{ Tratamento } & \multirow{2}{*}{$\begin{array}{c}\text { Cor vermelha } \\
(\%)\end{array}$} & \multicolumn{3}{|c|}{ Lado mais vermelho do fruto } & \multicolumn{3}{|c|}{ Lado menos vermelho do fruto } \\
\hline & & $L$ & $C$ & $h^{o}$ & $L$ & $C$ & $h^{o}$ \\
\hline & \multicolumn{7}{|c|}{ 'Catarina' } \\
\hline Controle & $59,5 b$ & $40,9 \mathrm{ab}$ & $31,9 b$ & $36,0 \mathrm{a}$ & $70,3 \mathrm{a}$ & $39,6 \mathrm{a}$ & $105,9 \mathrm{a}$ \\
\hline ProCa & $67,7 \mathrm{a}$ & $39,5 b$ & $30,8 b$ & $35,2 \mathrm{a}$ & $67,4 b$ & $38,8 \mathrm{a}$ & $102,1 \mathrm{ab}$ \\
\hline $\mathrm{GA}_{3}$ & $54,4 \mathrm{~b}$ & $42,9 \mathrm{a}$ & $33,6 \mathrm{a}$ & $38,0 \mathrm{a}$ & $70,9 \mathrm{a}$ & $39,5 \mathrm{a}$ & $101,5 b$ \\
\hline \multirow[t]{2}{*}{$\mathrm{CV}(\%)$} & 10,3 & 4,6 & 4,2 & 6,5 & 2,8 & 2,5 & 2,7 \\
\hline & \multicolumn{7}{|c|}{ 'Fuji' } \\
\hline Controle & $38,4 \mathrm{a}$ & $44,6 b$ & $26,5 b$ & $50,2 \mathrm{a}$ & $71,0 \mathrm{a}$ & $40,8 \mathrm{a}$ & $112,7 \mathrm{a}$ \\
\hline ProCa & $40,6 \mathrm{a}$ & $45,2 b$ & $25,7 b$ & $53,4 \mathrm{a}$ & $68,6 b$ & $42,0 \mathrm{a}$ & $113,1 \mathrm{a}$ \\
\hline $\mathrm{GA}_{3}$ & $41,1 \mathrm{a}$ & $47,8 \mathrm{a}$ & $29,7 \mathrm{a}$ & $54,7 \mathrm{a}$ & $71,1 \mathrm{a}$ & $41,7 \mathrm{a}$ & $109,5 b$ \\
\hline $\mathrm{CV}(\%)$ & 11,0 & 5,0 & 7,6 & 10,5 & 2,0 & 2,6 & 1,9 \\
\hline
\end{tabular}

Médias seguidas de mesma letra, nas colunas, para uma mesma cultivar, não diferem entre si, pelo teste de Tukey $(\mathrm{p}<0,05)$. 
TABELA 3- Índice de iodo-amido, sólidos solúveis (SS), acidez titulável (AT), firmeza de polpa e atributos de textura (forças para a penetração da casca e da polpa), no momento da colheita, em maçãs 'Fuji' e 'Catarina', provenientes de plantas submetidas aos tratamentos-controle, com prohexadiona-cálcio (ProCa) e giberelina $\left(\mathrm{GA}_{3}\right)$.

\begin{tabular}{|c|c|c|c|c|c|c|c|c|}
\hline \multirow{3}{*}{ Tratamento } & \multirow{3}{*}{$\begin{array}{l}\text { Índice } \\
\text { de iodo- } \\
\text { amido }\end{array}$} & \multirow{3}{*}{$\begin{array}{c}\mathrm{SS} \\
\left({ }^{\circ} \text { Brix }\right)\end{array}$} & \multirow{3}{*}{$\begin{array}{c}\text { AT } \\
(\mathrm{meq} \\
\left.100 \mathrm{~mL}^{-1}\right)\end{array}$} & \multirow{3}{*}{$\begin{array}{l}\text { Firmeza } \\
\text { de polpa } \\
\text { (N) }\end{array}$} & \multicolumn{4}{|c|}{--------------Atributos de textura ------------------ } \\
\hline & & & & & \multicolumn{2}{|c|}{$\begin{array}{c}\text { Força para ruptura } \\
\text { da epiderme }(\mathrm{N})\end{array}$} & \multicolumn{2}{|c|}{$\begin{array}{c}\text { Força para penetração } \\
\text { da polpa }(\mathrm{N})\end{array}$} \\
\hline & & & & & $\begin{array}{l}\text { Lado mais } \\
\text { vermelho }\end{array}$ & $\begin{array}{l}\text { Lado menos } \\
\text { vermelho }\end{array}$ & $\begin{array}{c}\text { Lado mais } \\
\text { vermelho }\end{array}$ & $\begin{array}{c}\text { Lado menos } \\
\text { vermelho }\end{array}$ \\
\hline & \multicolumn{8}{|c|}{ 'Catarina' } \\
\hline Controle & $2,2 \mathrm{a}$ & $11,3 \mathrm{a}$ & $0,50 \mathrm{a}$ & $89,4 \mathrm{a}$ & $16,3 \mathrm{ab}$ & $14,6 \mathrm{a}$ & $3,8 b$ & $3,6 a$ \\
\hline ProCa & $2,5 \mathrm{a}$ & $11,7 \mathrm{a}$ & $0,46 b$ & $91,5 \mathrm{a}$ & $16,6 \mathrm{a}$ & $14,8 \mathrm{a}$ & $4,1 \mathrm{a}$ & $3,7 \mathrm{a}$ \\
\hline $\mathrm{GA}_{3}$ & $2,6 a$ & $12,1 \mathrm{a}$ & $0,45 b$ & $88,4 \mathrm{a}$ & $15,2 b$ & $13,9 \mathrm{a}$ & $3,7 \mathrm{~b}$ & $3,6 \mathrm{a}$ \\
\hline \multirow[t]{2}{*}{$\mathrm{CV}^{3}(\%)$} & 14,4 & 5,0 & 8,2 & 3,1 & 5,2 & 4,9 & 6,6 & 3,5 \\
\hline & \multicolumn{8}{|c|}{ 'Fuji' } \\
\hline Controle & $4,1 \mathrm{a}$ & $11,0 \mathrm{~b}$ & $0,41 b$ & $79,2 \mathrm{ab}$ & $13,6 \mathrm{a}$ & $12,3 a$ & $3,1 b$ & $2,9 \mathrm{ab}$ \\
\hline ProCa & $4,0 \mathrm{a}$ & $10,9 b$ & $0,47 \mathrm{a}$ & $80,8 \mathrm{a}$ & $13,7 \mathrm{a}$ & $12,3 a$ & $3,4 a$ & $3,1 \mathrm{a}$ \\
\hline $\mathrm{GA}_{3}$ & $4,1 \mathrm{a}$ & $12,1 \mathrm{a}$ & $0,41 b$ & $75,8 b$ & $12,6 b$ & $11,6 b$ & $2,9 \mathrm{c}$ & $2,8 \mathrm{~b}$ \\
\hline $\mathrm{CV}^{3}(\%)$ & 6,1 & 5,8 & 8,0 & 3,9 & 4,7 & 4,0 & 7,7 & 5,9 \\
\hline
\end{tabular}

Médias seguidas de mesma letra, nas colunas, para uma mesma cultivar, não diferem entre si. pelo teste de Tukey $(\mathrm{p}<0,05)$.

TABELA 4- Sólidos solúveis (SS), acidez titulável (AT), firmeza de polpa e atributos de textura (forças para a penetração da casca e da polpa), após o armazenamento refrigerado, em maçãs 'Fuji' e 'Catarina' provenientes de plantas submetidas aos tratamentos-controle, com prohexadionacálcio (ProCa) e giberelina $\left(\mathrm{GA}_{3}\right)$.

\begin{tabular}{|c|c|c|c|c|c|c|c|}
\hline \multirow{3}{*}{ Tratamento } & \multirow{3}{*}{$\begin{array}{c}\mathrm{SS} \\
\left({ }^{\circ} \text { Brix }\right)\end{array}$} & \multirow{3}{*}{$\begin{array}{c}\text { AT } \\
(\mathrm{meq} \\
\left.100 \mathrm{~mL}^{-1}\right)\end{array}$} & \multirow{3}{*}{$\begin{array}{c}\text { Firmeza } \\
\text { de polpa } \\
\text { (N) }\end{array}$} & \multicolumn{4}{|c|}{---------------Atributos de textura --------------- } \\
\hline & & & & \multicolumn{2}{|c|}{$\begin{array}{c}\text { Força para ruptura da } \\
\text { epiderme }(\mathrm{N})\end{array}$} & \multicolumn{2}{|c|}{$\begin{array}{c}\text { Força para penetração } \\
\text { da polpa }(\mathrm{N})\end{array}$} \\
\hline & & & & $\begin{array}{l}\text { Lado mais } \\
\text { vermelho }\end{array}$ & $\begin{array}{c}\text { Lado menos } \\
\text { vermelho }\end{array}$ & $\begin{array}{c}\text { Lado mais } \\
\text { vermelho }\end{array}$ & $\begin{array}{c}\text { Lado menos } \\
\text { vermelho }\end{array}$ \\
\hline & \multicolumn{7}{|c|}{ 'Catarina' } \\
\hline Controle & $11,9 \mathrm{~b}$ & $0,14 \mathrm{a}$ & $78,0 \mathrm{~b}$ & $15,6 b$ & $14,2 \mathrm{ab}$ & $3,4 \mathrm{a}$ & $3,4 \mathrm{a}$ \\
\hline ProCa & $12,8 \mathrm{ab}$ & $0,14 \mathrm{a}$ & $88,2 \mathrm{a}$ & $16,6 \mathrm{a}$ & $14,7 \mathrm{a}$ & $3,7 \mathrm{a}$ & $3,4 \mathrm{a}$ \\
\hline $\mathrm{GA}_{3}$ & $13,5 \mathrm{a}$ & $0,15 \mathrm{a}$ & $79,8 b$ & $15,0 \mathrm{~b}$ & $13,6 b$ & $3,5 \mathrm{a}$ & $3,3 \mathrm{a}$ \\
\hline $\mathrm{CV}(\%)$ & 10,2 & 15,8 & 6,6 & 6,2 & 5,7 & 7,5 & 6,0 \\
\hline \multicolumn{8}{|c|}{ 'Fuji' } \\
\hline Controle & $11,1 b$ & $0,11 \mathrm{ab}$ & $66,4 \mathrm{a}$ & $12,6 \mathrm{a}$ & $11,3 a b$ & $3,1 \mathrm{a}$ & $2,8 \mathrm{a}$ \\
\hline ProCa & $11,2 b$ & $0,12 \mathrm{a}$ & $67,3 \mathrm{a}$ & $12,9 a$ & $11,7 \mathrm{a}$ & $3,1 \mathrm{a}$ & $2,9 \mathrm{a}$ \\
\hline $\mathrm{GA}_{3}$ & $11,7 \mathrm{a}$ & $0,10 \mathrm{~b}$ & $63,3 b$ & $10,3 b$ & $10,8 b$ & $2,8 \mathrm{~b}$ & $2,9 a$ \\
\hline $\mathrm{CV}^{3}(\%)$ & 5,3 & 14,4 & 4,1 & 11,2 & 6,6 & 7,6 & 5,9 \\
\hline
\end{tabular}

Médias seguidas de mesma letra, nas colunas, para uma mesma cultivar, não diferem entre si, pelo teste de Tukey $(\mathrm{p}<0,05)$. 


\section{CONCLUSÕES}

A pulverização de macieiras em pós-floração com $\mathrm{GA}_{3}\left(319 \mathrm{~g} \mathrm{ha}^{-1}\right)$ pode comprometer a floração no ano subsequente à sua aplicação e alterar alguns atributos de qualidade dos frutos, indicando avanço na maturação. A pulverização em pós-floração com ProCa (319 $\left.\mathrm{g} \mathrm{ha}^{-1}\right)$ pode ser uma alternativa para aumentar a consistência de polpa e a porcentagem de cor vermelha em frutos de macieiras 'Catarina'. Entretanto, este tratamento pode reduzir a floração e a frutificação no ano subsequente a sua aplicação em macieiras 'Fuji'.

\section{AGRADECIMENTOS}

Ao CNPq e à CAPES, pela concessão de bolsas; à FAPESC, pelo apoio financeiro, e à EPAGRI de São Joaquim-SC, por ceder a área experimental para a execução deste projeto.

\section{REFERÊNCIAS}

AMARANTE, C.V.T.; DREHMER, A.M.F.; SOUZA, F.; FRANCESCATTO, P. A pulverização pré-colheita com ácido giberélico $\left(\mathrm{GA}_{3}\right)$ e aminoetoxivinilglicina (AVG) retarda a maturação e reduz as perdas de frutos na cultura do pessegueiro. Revista Brasileira de Fruticultura, Jaboticabal, v.27, n.1, p.1-5, 2005.

AMARANTE, C.V.T.; STEFFENS, C.A.; BLUM, L.E.B. Coloração do fruto, distúrbios fisiológicos e doenças em maçãs 'Gala' e 'Fuji' pulverizadas com aminoetoxivinilglicina. Revista Brasileira de Fruticultura, Jaboticabal, v.32, n.1, p.9-18, 2010.

AMARANTE, C.V.T.; STEFFENS, C.A.; MOTA, C.S.; SANTOS, H.P. Radiação, fotossíntese, rendimento e qualidade de frutos em macieiras 'Royal Gala' cobertas com telas antigranizo. Pesquisa Agropecuária Brasileira, Brasília, v.42, n.7, p.925-931, 2007.

BASAK, A. Growth and fruiting of 'Elstar' apple trees in response to prohexadione calcium depending on the rootstock. Acta Horticulturae, The Hague, n.653, p.117-126, 2004.

COSTA, G.; SABATINI, F.; SPINELLI, F.; ANDREOTTI, C.; BOMBEN, C.; VIZZOTO, G. Two years of application of prohexadione-Ca on apple: effect on vegetative and cropping performance, fruit quality, return bloom and residual effect. Acta Horticulturae, The Hague, n.653, p.27-132, 2004.
DAVIS, D.E. Inhibition of flower bud initiation and development in apple by defoliation, gibberellic acid and crop load manipulation. 2002. 120 f. Thesis (Doctor of Philosophy) - Faculty of Virginia Polytechnic Institute and State University, Blacksburg, 2002.

DE GRAUWE, L.; DUGARDEYN, J.; STRAETEN, D.V.D. Novel mechanisms of ethylene-gibberellin crosstalk revealed by the gai eto2-1 double mutant. Plant Signaling \& Behavior, New Delhi, v.3, n.12, p.1113-1115, 2008.

FERGUSON, I.B.; SNELGAR, W.; BOWEN, J.H.; WOOLF, A.B. Preharvest field heat and postharvest fruit response. Acta Horticulturae, The Hague, n.485, p.149-154, 1999.

FREITAS, S.T.;AMARANTE, C.V.T.; LABAVITCH, J.M.; MITCHAM, E.J. Cellular approach to understand bitter pit development in apple fruit. Postharvest Biology and Technology, Amsterdam, v.57, n.1, p.6-13, 2010.

GOTTGENS, E.F.; HEDDEN, P. Gibberellin as a factor in floral regulatory networks. Journal of Experimental Botany, Oxford, v.60, n.7, p.19791989, 2009.

GREENE, D.W. The effect of repeat annual applications of prohexadione-calcium on fruit set, return bloom, and fruit size of apples. HortScience, Alexandria, v.43, n.2, p.376-379, 2008.

GREENE, D.W. Tree growth management and fruit quality of apple trees treated with prohexadionecalcium (BAS 125). HortScience, Alexandria, v.34, n.7, p.1209-1212, 1999.

HAWERROTH, J.F.; PETRI, J.L.; FACHINELLO, J.C.; HERTER, F.G.; PREZOTTO, M.E.; HASS, L.B.; PRETTO, A. Redução da poda hibernal e aumento da produção de pereiras 'Hosui' pelo uso de prohexadiona cálcio. Pesquisa Agropecuária Brasileira, Brasília, v.47, n.7, p.939-947, 2012 b.

HAWERROTH, J.F.; PETRI, J.L.; LEITE, G.B.; YOSHIKAWA, E.R. Épocas de aplicação de proexadione cálcio no controle do desenvolvimento vegetativo de macieiras 'Imperial Gala'. Revista Brasileira de Fruticultura, Jaboticabal, v.34, n.4, p.957-963, 2012a. 
KHURSHID, T.; McNEIL, D.L.; TROUGHT, M.C.T.; HILL, G.D. The response of young 'Braeburn' and 'Oregon Spur Delicious' apple trees growing under an ultrahigh density planting system to soil-applied paclobutrazol. II Effect on fruit quality at harvest and during storage. Scientia Horticulturae, Leuven, v.71, p.189-196, 1997.

MEDJDOUB, R.; VAL, J.; BLANKO, A. Inhibition of vegetative growth in red apple cultivars using prohexadione-calcium. Journal of Horticultural Science and Biotechnology, Clinton, v.80, n.2, p.263-271, 2005.

PEREIRA, L.B.; SIMIONI, F.J.; CARIO, S.A.F. Evolução da produção de maçã em Santa Catarina: novas estratégias em busca de maior competitividade. Revista Ensaios FEE, Porto Alegre, v.31, n.1, p.209234, 2010.

RAMÍREZ, H.; MANJARREZ, R.M.P.; BENAVIDES-MENDONZA, A.; SANCHEZLÓPES, A.; TORRES, V.R.; DAVILA, J.H. Efectos de prohexadione-Ca em tomate y su relación com la variación de la concentración de giberelinas y citocininas. Revista Chapingo - Série Horticultura, Chapingo, v.11, n.2, p.283-290, 2005.

SAS INSTITUTE. Getting started with the SAS learning edition. Cary, 2002. 200p.

SCHMIDT, T.R.; ELFVING, D.C.; McFERSON, J.R.; WHITING, M.D. Gibberellic acid accelerates 'Honeycrisp', but not 'Cameo', apple fruit maturation. HortTechnology, Chicago, v.18, n.1, p.39-44, 2008.
SILVEIRA, J.P.G.;AMARANTE, C.V.T.; STEFFENS, C.A.; MIQUELOTO, A.; KATSURAYAMA, J.M. A inibição na síntese de giberelina reduz o crescimento vegetativo em macieiras e proporciona controle de "bitter pit" nos frutos. Revista Brasileira de Fruticultura, Jaboticabal, v.34, n.2, p. 328-335, 2012.

STEFFENS, C.A.; AMARANTE, C.V.T.; CHECHI, R.; SILVEIRA, J.P.G.; BRACKMANN, A. Aplicação pré-colheita de reguladores vegetais visando a retardar a maturação de ameixas 'Laetitia'. Ciência Rural, Santa Maria, v.39, n.5, p.1.369-1.373, 2009.

WILKIE, J.D.; SEDGlEY, M.; OLESEN, T. Regulation of floral initiation in horticultural trees. Journal of Experimental Botany, Oxford, v.59, n.12, p.3215-3228, 2008.

YESHITELA, T.; ROBBERTSE, P.J.; STASSEN, P.J.C. Paclobutrazol suppressed vegetative growth and improved yield as well as fruit quality of 'Tommy Atkins' mango (Mangifera indica) in Ethiopia. New Zealand Journal of Crop and Horticultural Science, Wellington, v.32, p.281-293, 2004.

ZHANG, C.; LEE, U.; TANABE, K. Hormonal regulation of fruit set, parthenogenesis induction and fruit expansion in Japanese pear. Plant Growth Regulation, New York, v.55, n.3, p.231-240, 2008.

ZHANG, C.; WHITING, M. Pre-harvest foliar application of prohexadione-Ca and gibberellins modify canopy source-sink relations and improve quality and shelf-life of 'Bing' sweet cherry. Plant Growth Regulation, New York, v.65, n.1, p.145156, 2011. 